\title{
Implementation of a spatial two-dimensional quantum random walk with tunable decoherence
}

\author{
J. Svozilík, ${ }^{1,2}$ R. de J. León-Montiel, ${ }^{1}$ and J. P. Torres ${ }^{1,3}$ \\ ${ }^{1}$ ICFO-Institut de Ciencies Fotoniques, Mediterranean Technology Park, 08860 Castelldefels, Barcelona, Spain \\ ${ }^{2}$ Palacký University, RCPTM, Joint Laboratory of Optics, 17. listopadu 12, 77146 Olomouc, Czech Republic \\ ${ }^{3}$ Department of Signal Theory and Communications, Universitat Politècnica de Catalunya, Castelldefels, O8860 Barcelona, Spain
}

(Received 17 July 2012; published 26 November 2012)

\begin{abstract}
We put forward a versatile and highly scalable experimental setup for the realization of discrete two-dimensional quantum random walks with a single-qubit coin and tunable degree of decoherence. The proposed scheme makes use of a small number of simple optical components arranged in a multipath Mach-Zehnder-like configuration, where a weak coherent state is injected. Environmental effects (decoherence) are generated by a spatial light modulator, which introduces pure dephasing in the transverse spatial plane perpendicular to the direction of propagation of the light beam. By controlling the characteristics of this dephasing, one can explore a great variety of scenarios of quantum random walks: pure quantum evolution (ballistic spread), fast fluctuating environment leading to a diffusive classical random walk, and static disorder resulting in the observation of Anderson localization.
\end{abstract}

DOI: 10.1103/PhysRevA.86.052327

PACS number(s): 03.67.Ac, 03.65.Yz

\section{INTRODUCTION}

The underlying principles of many quantum information protocols can be traced back to the concept of quantum random walk (QRW), which since its first description has become a fundamental paradigm in quantum science $[1,2]$. The idea of QRWs was originally conceived by Aharonov et al. [3] as an extension of the well-known classical random walk (CRW) [4]. The main distinguishing feature of a QRW, compared to a $\mathrm{CRW}$, is the possibility of interference between the multiple paths that can be simultaneously traversed by a quantum walker, thus enabling a faster spreading of the uncertainty of location of the walker than in the classical case $[5,6]$.

The temporal evolution of a quantum system, such as a QRW, depends on the presence and specific characteristics of the environmental effects (decoherence) which can modify it [7]. In most cases, the influence of decoherence during the evolution of a quantum walker transforms an originally pure state into a mixed state, lowering the uncertainty about the location of the walker as it propagates. In the limiting case, when all cross-interference terms between different lattice sites are completely erased, the state of pure diffusive classical propagation is reached [6].

QRWs have been theoretically explored for the case of onedimensional lattices $[5,8]$ and experimentally implemented by means of different physical platforms, such as photon-based systems [9-14], optical lattices [15], and waveguide arrays [16]. Also, QRWs have been implemented using trapped ions [17] and nuclear magnetic resonance systems [18].

Although the implementation of one-dimensional QRWs has been useful when describing several quantum information systems, there is a great interest in expanding the concept to multidimensional lattices. Along these lines, two-dimensional QRWs provide a powerful tool for modeling complex quantum information and energy transport systems [19,20]. Their realization presents a challenge because of the need for four-level coin operation [21-23]. One way to overcome this drawback is to make use of different degrees of freedom of photons, such as polarization and orbital angular momentum, as has been shown in Ref. [20]. Another approach is to mimic the two-dimensional QRW evolution by performing two subsequent one-dimensional QRWs [24,25].

Here, we make use of the latter approach to put forward an experimental setup for the realization of two-dimensional QRWs. We include the environmental effects (decoherence) as pure dephasing by means of the introduction of random phase patterns, generated by a spatial light modulator (SLM), which can be different from site to site (spatial disorder). By controlling the degree of decoherence, we study the transition from the quantum ballistic spreading to the diffusive classical walk. Also, by adding static disorder, we show the possibility of observing Anderson localization [26].

Importantly, our proposal provides a versatile, highly scalable experimental setup, which may be used as a tool for understanding quantum processes whose underlying physics can be somehow traced to the concept of random walks, such as energy transport in photosynthetic light-harvesting complexes [27,28] and material band-gap structures [29].

The structure of the article is as follows. In Sec. II, we introduce the QRW model system, including dephasing, considered here. The proposed experimental setup is described in Sec. III. Numerical results are discussed in Sec. IV. Finally, we summarize our results in the conclusion.

\section{A TWO-DIMENSIONAL QUANTUM RANDOM WALK WITH DEPHASING}

A typical discrete quantum random walk comprises two operations: a coin-tossing operation and a shift operation. Here, the coin-tossing operation is performed in the Hilbert space $\mathcal{H}_{p}$ spanned by vectors $\{|H\rangle,|V\rangle\}$, corresponding to the photon polarization. The random walk is performed in the Hilbert space $\mathcal{H}_{X} \otimes \mathcal{H}_{Y}$, corresponding to the position of the photon in the transverse plane, spanned by vectors $\{|i, j\rangle\}(i, j$ integers), which indicate sites $(i, j)$ in the transverse plane $(i, j=\ldots,-2,-1,0,1,2, \ldots)$. The global quantum system thus evolves in the Hilbert space

$$
\mathcal{H}=\mathcal{H}_{X} \otimes \mathcal{H}_{Y} \otimes \mathcal{H}_{p}
$$


The state of the system is described by the density matrix $\hat{\rho}^{(n)}$, which is transformed to a new density matrix each step $n$ via the map

$$
\hat{\rho}^{(n+1)}=\hat{P}^{(n)} \hat{S}_{Y} \hat{H} \hat{S}_{X} \hat{H} \hat{\rho}^{(n)} \hat{H}^{\dagger} \hat{S}_{X}^{\dagger} \hat{H}^{\dagger} \hat{S}_{Y}^{\dagger} \hat{P}^{(n) \dagger} .
$$

$\hat{H}$ denotes the Hadamard operator

$$
\hat{H}=\frac{1}{\sqrt{2}}\left(\begin{array}{cc}
1 & 1 \\
1 & -1
\end{array}\right),
$$

which acts on the polarization degree of freedom. The operators $\hat{S}_{X}$ and $\hat{S}_{Y}$, which describe the walker's shift in the transverse dimensions $X$ and $Y$, independently, read as

$$
\hat{S}_{X}=\sum_{i, j}|i-1, j, H\rangle\langle i, j, H|+| i+1, j, V\rangle\langle i, j, V|
$$

and

$$
\hat{S}_{Y}=\sum_{i, j}|i, j-1, H\rangle\langle i, j, H|+| i, j+1, V\rangle\langle i, j, V| .
$$

The coupling of the quantum walker with the environment is described by pure dephasing [30,31]. The form of the unitary dephasing operator considered here can be written as

$$
\hat{P}^{(n)}=\sum_{i, j} e^{-\frac{i}{2} \phi_{i j}^{(n)} \hat{\sigma}_{z}}|i, j\rangle\langle i, j|
$$

where $\phi_{i j}^{(n)}$ is a random phase matrix and $\hat{\sigma}_{z}$ is the Pauli operator. Inspection of Eq. (6) shows that $\phi_{i j}^{(n)}$ represents a newly introduced phase difference between the horizontal and vertical polarizations at each site. We consider three physically relevant scenarios that can be easily implemented in the setup proposed here (see Sec. III). In the general case, the phase differences $\phi_{i j}^{(n)}$ are independent random variables, but with the same probability distribution. Moreover, the ensemble of phase differences $\phi_{i j}^{(n)}$ can change from step $n$ to step $n+1$. In the following, we refer to this case as a QRW influenced by dynamical spatial disorder.

The easiest probability distribution that we can consider is an uniform probability distribution. If phases can be chosen arbitrarily between the extreme values $-\zeta$ and $\zeta$, there is a constant probability $1 /(2 \zeta)$ of obtaining any phase in this interval. $\zeta=\pi$ is the maximal phase which we can have between the two orthogonal polarizations. $\zeta=0$ corresponds to the absence of any spatial disorder. If phases do not change during propagation, even though they might differ from site to site (i.e., $\phi_{i j}^{(n)}=\phi_{i j}^{(n+1)}$ ), then we have static spatial disorder. Finally, if all phase differences are the same for all sites, but they can still change from one step to the following, we have dynamical dephasing without spatial disorder.

The probability of detecting a photon in the site $(i, j)$ is

$$
p^{(n)}(i, j)=\left\langle i, j\left|\operatorname{Tr}_{p}\left[\hat{\rho}^{(n)}\right]\right| i, j\right\rangle,
$$

where the density matrix that describes the whole system is traced out over the polarization degree of freedom $\left(\operatorname{Tr}_{p}\right)$.

The spreading of the uncertainty of photon location is characterized by dependence of the variance on the step index $n$

$$
V^{(n)}=\sum_{i, j} p^{(n)}(i, j)\left|\mathbf{r}_{i j}-\mu\right|^{2}
$$

where $\mathbf{r}_{i j}=(i, j)$ represents the lattice site with indexes $(i, j)$ and $\mu$ is the mean position; that is, $\mu=\sum_{i, j} p^{(n)}(i, j) \mathbf{r}_{i j}$.

\section{EXPERIMENTAL SETUP}

The main building block of the QRW setup is the multipath Mach-Zehnder-like configuration shown in Fig. 1. It allows us to make several runs of the QRW without the necessity of using a large amount of optical components, as it is the case, for instance, of the experiment described in Ref. [10]. A similar scheme, based on a single-path Mach-Zehnder-like configuration, has been used [11,14]. However, in these cases, the walker moves in time, whereas in our proposal the walker moves in the two-dimensional transverse plane, offering a way to simplify the experimental implementation of the two-dimensional QRW.

As source of photons one can use a highly attenuated short coherent pulse, prepared by the combination of a photon source and attenuator (AT), generating the initial state

$$
\left|\Psi^{(0)}\right\rangle=|0,0\rangle \otimes \frac{1}{\sqrt{2}}(|H\rangle+i|V\rangle),
$$

where $(0,0)$ is the central site. The duration of the pulse has to be sufficiently smaller than the time of flight through the setup in one cycle. The transverse size of the Gaussian beam profile of the pulse has to be carefully chosen, so that two adjacent sites are not overlapping in the space due to diffraction. For instance, by making use of a Gaussian beam of 2-mm beam waist, corresponding to a Rayleigh range of $23.6 \mathrm{~m}$ (for a wavelength $\lambda=532 \mathrm{~nm}$ ), along with typical-sized optical components, we could in principle perform a QRW of approximately more than 20 steps. The number of steps can be further improved by applying smaller beams together with a refocusing system placed along the walker's path. Alternatively, a spontaneous parametric down-conversion source can be used, provided each

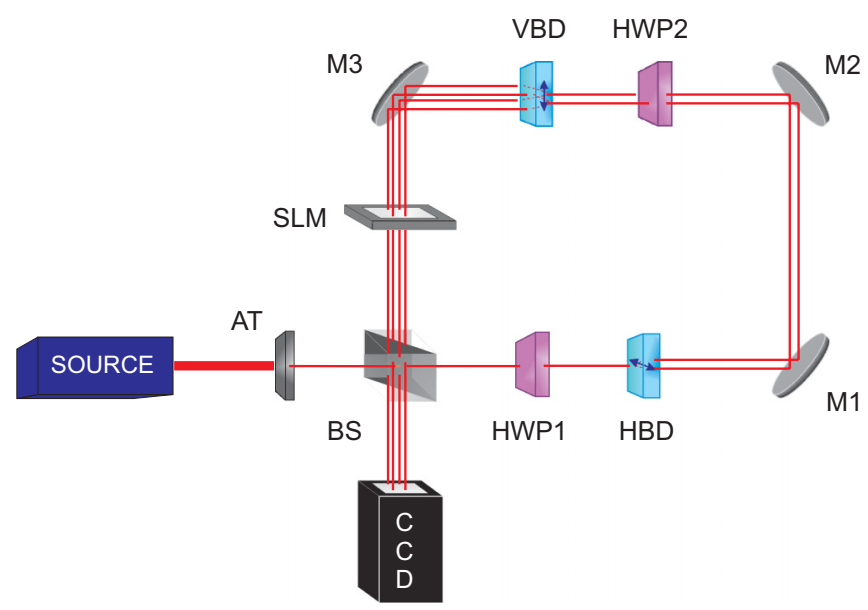

FIG. 1. (Color online) General scheme for the implementation of a two-dimensional random walk with decoherence. AT, attenuator; BS, beam splitter; HWP1 and HWP2, half-wave plates that act as coins in the random walk; HBD and VBD, horizontal and vertical beam displacers; M1, M2, and M3, mirrors; SLM, spatial light modulator; and $\mathrm{CCD}$, spatial light sensor with single-photon sensitivity. 
down-converted photon is generated in a pure state. Then, the photon is transmitted via the beam splitter (BS) to the system.

To get a clearer picture of the working of the quantum random walk, let us consider in detail the quantum state of the photon in its first passage through the system. First, the polarization state of the photon is changed by the half-wave plate (HWP1) to $\frac{1}{2}|0,0\rangle \otimes[(1+i)|H\rangle+(1-i)|V\rangle]$; that is, the Hadamard $(\hat{H})$ operation is applied. After this transformation, the photon is displaced by the horizontal beam displacer (HBD) along the $X$ axis according to its polarization, as described by the shift operator $\hat{S}_{X}$. The photon is now in the state $\frac{1}{2}[(1+i)|-1,0, H\rangle+(1-i)|1,0, V\rangle]$. A second half-wave plate (HWP2) implements a new Hadamard transformation, which transforms the quantum state to $\frac{1}{\sqrt{8}}\{(1+i)(|-1,0, H\rangle+|-1,0, V\rangle)+(1-i)(|1,0, H\rangle-$ $|1,0, V\rangle)\}$. The vertical beam displacer (VBD) shifts the position of the photon along the $Y$ axis. After this, the quantum state of the photon reads

$$
\begin{aligned}
\left|\Psi^{(1)}\right\rangle= & \frac{1}{\sqrt{8}}[(1+i)(|-1,-1, H\rangle+|-1,1, V\rangle) \\
& +(1-i)(|1,-1, H\rangle-|1,1, V\rangle)] .
\end{aligned}
$$

The spatial light modulator (SLM) is used to introduce random phases, given by the phase-matrix $\phi_{i j}^{(n)}$. After the photon passes through the SLM, the whole cycle is repeated with high probability. If not, the photon escapes through the other output port of the beam splitter, which directs it towards a single-photon sensitive charge-coupled device (CCD) camera with an integrated electron multiplier, which allows us to spatially resolve a weak signal with a high efficiency ( $90 \%)$ [32]. Moreover, losses in the setup can be compensated by an increase of the amplitude of pulse. The size of whole array of beams can be reduced by an auxiliary imaging system in order to fit on the limited size of the sensitive area of the CCD.

\section{A. Fast exchange of phase matrices}

A typical SLM has a response time in the order of tens of milliseconds, which means that it is too slow for a fast phasemask exchange. For this reason, the transmission SLM shown in Fig. 1 has to be supplemented by additional components as shown in Fig. 2, which allows us to effectively generate the dynamical spatial disorder and dynamical dephasing without spatial disorder for a limited amount of steps. The time of

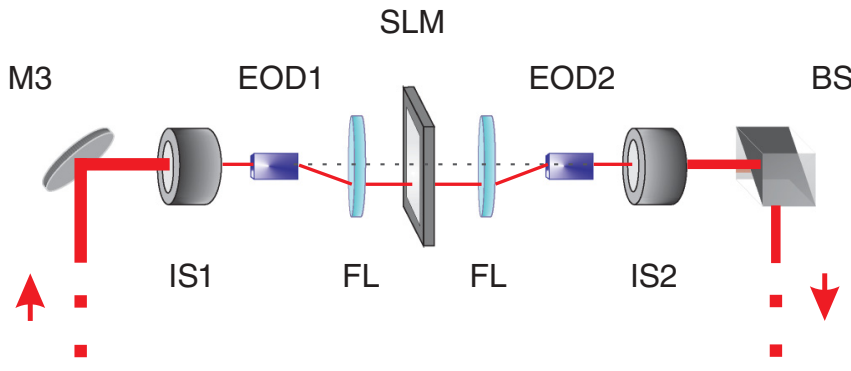

FIG. 2. (Color online) Detail of the SLM part of the setup allowing generation of dynamical spatial disorder. M3, mirror; EOD1 and EOD2, electro-optic deflectors; FL, Fourier lens; SLM, spatial light modulator; BS, beam splitter; and IS1 and IS2, imaging systems exchange of phase matrices can be done now in tens of picoseconds [33,34], which is three orders faster than necessary in our experimental proposal, with a typical time of flight of the pulse in the order of nanoseconds. The scheme in Fig. 2 operates in the following manner. At the beginning, the size of the whole array of beams is reduced by an imaging system (IS1) to fit into the electro-optical deflector (EOD1). The EOD1, together with a Fourier lens (FL), serves to address different regions of the SLM along either the vertical or horizontal directions, which realize then the random-phase matrices $\phi_{i j}^{(n)}$ in all steps. By changing the directions of deflection of the array, both types of dynamical disorders can be simulated. The EOD2 (and another FL) then serve to return all beams back to the original direction of propagation, so that all beams remain in the same position in the traverse plane at all time.

\section{NUMERICAL RESULTS}

\section{A. Quantum random walk}

Let us consider first the case when the SLM does not introduce any phase shift $\left[\phi_{i j}^{(n)}=0\right.$ for all $\left.(i, j)\right]$. This corresponds to the case $\zeta=0$. Figure 3 shows the probability distribution function $p^{(n)}(i, j)$ for (a) $n=10$ and (b) $n=20$ steps. In both cases, the distribution shows a symmetrical shape around the lines $X=0$ and $Y=0$, with four groups of peaks located along the $X$ and $Y$ axes. The resulting symmetry comes from the specific initial quantum state chosen in Eq. (9). When the number of steps is increased, the peaks move


FIG. 3. (Color online) Probability distribution function $p^{(n)}(i, j)$, corresponding to the position of the photon for a two-dimensional quantum random walk with no dephasing after (a) 10 steps and (b) 20 steps. 

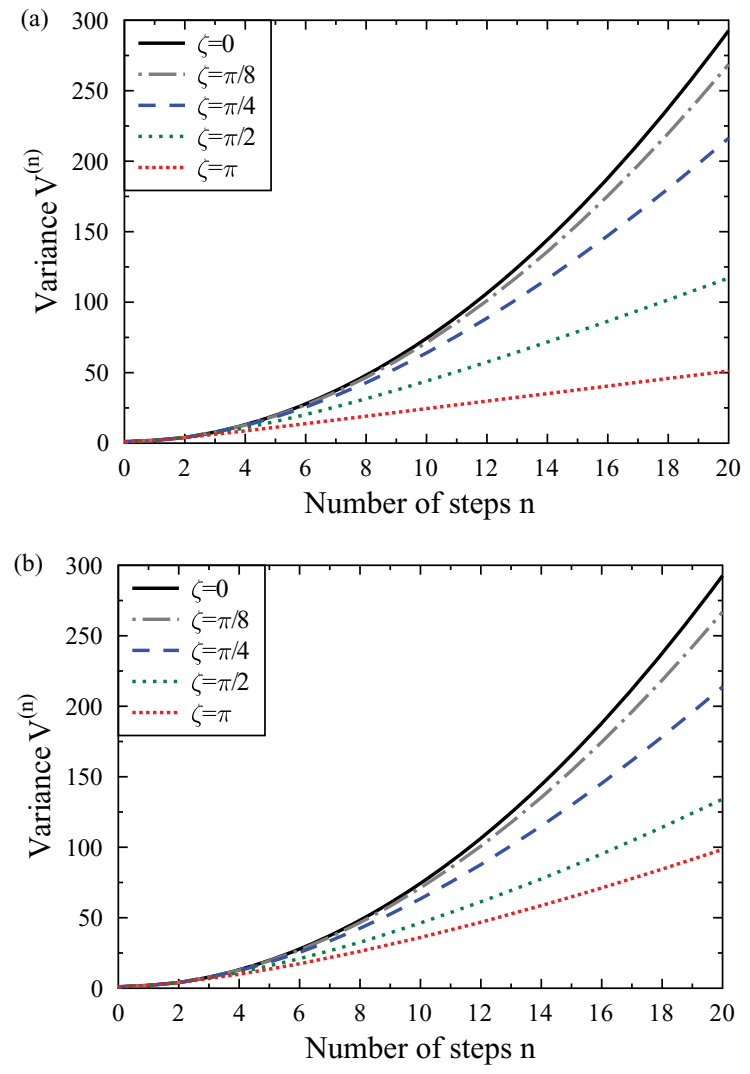

FIG. 4. (Color online) Spreading of the position of the photon $\left(V^{(n)}\right)$ as a function of the number of steps for several values of $\zeta$ and different types of dephasing. (a) Dynamical spatial disorder. (b) Dynamic dephasing without spatial disorder. The results are obtained by averaging over 500 different realizations of the $\operatorname{matrix} \phi_{i j}^{(n)}$.

further away from the central site $(0,0)$. The shapes obtained in Fig. 3 correspond to the probability distributions of a two-dimensional Grover walk $[19,25]$. The walker propagates with ballistic speed, characterized by a quadratic dependence of the variance $V^{(n)}$ with the step index, that is, $V^{(n)} \approx n^{2}$. This case is shown in Figs. 4(a) and 4(b), corresponding to the case with $\zeta=0$.

\section{B. Quantum random walk affected by dephasing}

The dephasing effect introduced by the SLM allows us to induce a transition from the quantum to the classical random walk via two mechanisms. The first, as shown in Fig. 4(a), is by means of dynamical spatial disorder. The phase matrix $\phi_{i j}^{(n)}$ shows independent and randomly chosen values for each site, and it is refreshed each step. The case $\zeta=0$ corresponds to the QRW with no dephasing. Increasing the amount of disorder, characterized by a corresponding increase of the parameter $\zeta$, reduces the spreading of $V^{(n)}$ as can be seen in Fig. 4(a). In the limiting case, which is reached for $\zeta=\pi$, the observed dependence $(\sim n)$ of variance $V^{(n)}$ is a direct indication of the transition to the classical regime of random walks.

The classical limit can also be reached by means of dynamical dephasing without spatial disorder, as shown in Fig. 4(b). The reduction of the uncertainty of the photon
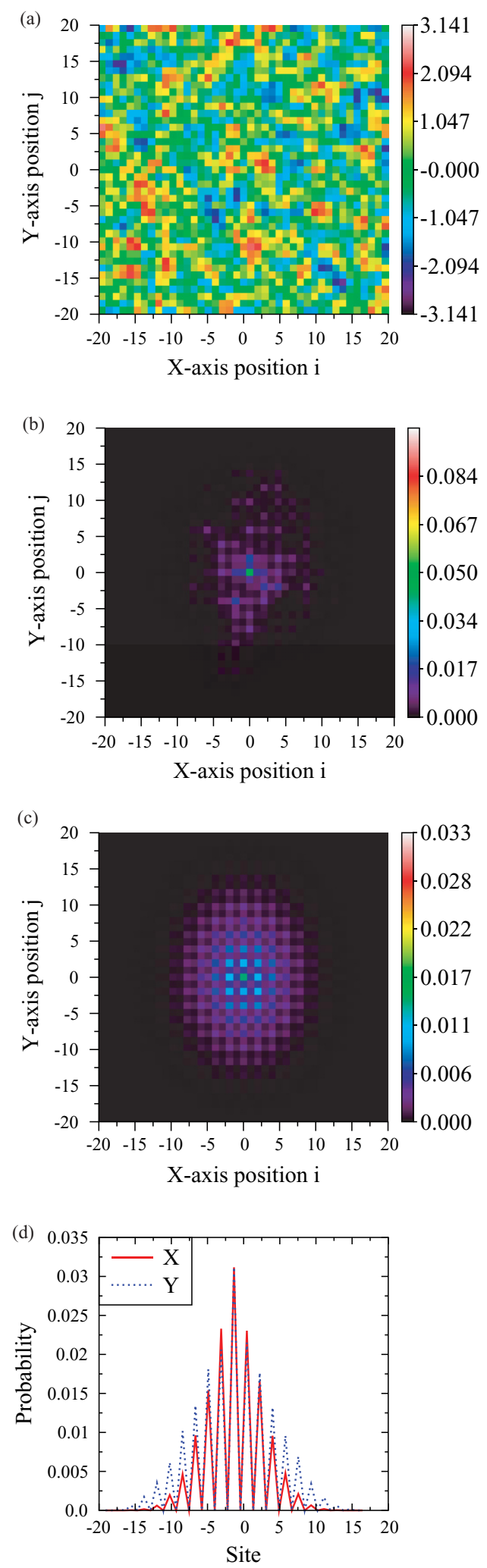

FIG. 5. (Color online) Observation of the spatial Anderson localization. (a) Example of a matrix $\phi_{i j}^{(n)}$ for $\zeta=\pi$ that leads to the Anderson localization. (b) Corresponding probability distribution $p^{(n)}(i, j)$ after 20 steps. (c) Averaged probability distribution over 500 realizations. (d) Cuts of the data shown in Fig. 5(c) along the $X$ and $Y$ axes passing the site $(0,0)$. 
position is less dramatic than in the case with dynamical spatial disorder. For the dynamical spatial disorder, $V^{(n)} \sim 51.25$ for $\zeta=\pi$ after 20 steps. On the contrary, for dynamical dephasing without spatial disorder, we have $V^{(n)} \sim 98.45$ under the same conditions. Indeed, the $n$ dependence of the typical deviation, characteristic of the classical regime, it is not yet reached after 20 steps, as is readily observed in Fig. 4(b).

\section{Anderson localization}

In the context of our discussion, the Anderson localization is the reduction of spreading of the uncertainty of the photon position [26]. We demonstrate that this effect can also be observed in the setup considered here. In Ref. [35], Anderson localization was observed in the transverse plane of a light beam passing through a crystal with random static fluctuations of the index of refraction. Since the randomness in the index of refraction is affecting only the phase of the propagating beam, it is possible to imitate these phase fluctuations with a SLM, under the consideration of static spatial disorder, since Anderson localization does not appear with dynamical spatial disorder.

In Fig. 5(a) we present a typical profile of the phase matrix $\phi_{i j}^{(n)}$, independent of $n$, which leads to beam localization. Figure 5(b) shows the corresponding probability distribution of the photon position for this specific phase profile. Notice that it contains a strong peak located in the middle of the lattice. The presence of Anderson localization is confirmed in Fig. 5(c), where we show the averaged probability distribution function for $\zeta=\pi$, exhibiting an exponential suppression of probabilities for sites distant from the center. For the sake of clarity, we also plotted in Fig. 5(d) two cuts of the averaged probability distribution along the $X$ and $Y$ axes, to highlight this feature.

\section{CONCLUSION}

We have put forward a new, highly scalable, and easily implemented experimental configuration to observe spatial two-dimensional random walks under a great variety of circumstances by means of the implementation of two consecutive one-dimensional random walks. The proposal makes use of only a small amount of simple optics components and allows us to simulate many different quantum systems and protocols based on the quantum random walk concept. Additionally, by carefully controlling the amount and type of disorder present in the system, we have shown the effects of different environmental effects: dynamical spatial disorder, dynamical dephasing without spatial disorder, and static spatial disorder. The last case drove us to the observation of Anderson localization. The control of environmental effects is of paramount importance in nearly all quantum systems. In some cases, it is even crucial to understanding the dynamics experimentally observed. For instance, in light-harvesting complexes [36], the interplay between coherent evolution and noise is a critical ingredient, which allows high-efficiency energy transport.

\section{ACKNOWLEDGMENTS}

The authors thank Jan Soubusta for useful discussions. This work was supported by Projects No. FIS2010-14831 and No. FET-Open 255914 (PHORBITECH). J.Sv. acknowledges Project FI-DGR 2011 of the Catalan government. This work was also supported in part by Projects COST OC 09026 and CZ.1.05/2.1.00/03.0058 of the Ministry of Education, Youth, and Sports of the Czech Republic and by Project PrF-2012-003 of Palacký University.
[1] B. L. Douglas and J. B. Wang, Phys. Rev. A 79, 052335 (2009).

[2] N. Shenvi, J. Kempe, and K. Birgitta Whaley, Phys. Rev. A 67, 052307 (2003).

[3] Y. Aharonov, L. Davidovich, and N. Zagury, Phys. Rev. A 48, 1687 (1993).

[4] N. G. van Kampen, Stochastic Processes in Physics and Chemistry (North Holland, Amsterdam, 2007).

[5] J. Kempe, Contemp. Phys. 44, 307 (2003).

[6] V. Kendon, Math. Struct. Comp. Sci. 17, 1169 (2006).

[7] M. Schlosshauer, Decoherence and the Quantum-to-Classical Transition (Springer, Heidelberg/Berlin, 2007).

[8] A. Ambainis, E. Bach, A. Nayak, A. Vishwanath, and J. Watrous, in Proceedings of the Thirty-Third Annual ACM Symposium on Theory of Computing (ACM, New York, 2001).

[9] A. Peruzzo, M. Lobino, J. C. F. Matthews, N. Matsuda, A. Politi, K. Poulios, X.-Q. Zhou, Y. Lahini, N. Ismail, K. Wörhoff, Y. Bromberg, Y. Silberberg, M. G. Thompson, and J. L. OBrien, Science 329, 1500 (2010).

[10] M. A. Broome, A. Fedrizzi, B. P. Lanyon, I. Kassal, A. Aspuru-Guzik, and A. G. White, Phys. Rev. Lett. 104, 153602 (2010).
[11] A. Schreiber, K. N. Cassemiro, V. Potoček, A. Gábris, P. J. Mosley, E. Andersson, I. Jex, and Ch. Silberhorn, Phys. Rev. Lett. 104, 050502 (2010).

[12] D. Pandey, N. Satapathy, M. S. Meena, and H. Ramachandran, Phys. Rev. A 84, 042322 (2011).

[13] L. Sansoni, F. Sciarrino, G. Vallone, P. Mataloni, A. Crespi, R. Ramponi, and R. Osellame, Phys. Rev. Lett. 108, 010502 (2012).

[14] A. Schreiber, K. N. Cassemiro, V. Potoček, A. Gabris, I. Jex, and Ch. Silberhorn, Phys. Rev. Lett. 106, 180403 (2011).

[15] R. Côté, A. Russell, E. E. Eyler, and P. L. Gould, New J. Phys. 8, 156 (2006).

[16] H. B. Perets, Y. Lahini, F. Pozzi, M. Sorel, R. Morandotti, and Y. Silberberg, Phys. Rev. Lett. 100, 170506 (2008).

[17] F. Zähringer, G. Kirchmair, R. Gerritsma, E. Solano, R. Blatt, and C. F. Roos, Phys. Rev. Lett. 104, 100503 (2010).

[18] J. Du, H. Li, X. Xu, M. Shi, J. Wu, X. Zhou, and R. Han, Phys. Rev. A 67, 042316 (2003).

[19] A. C. Oliveira, R. Portugal, and R. Donangelo, Phys. Rev. A 74, 012312 (2006).

[20] A. Schreiber, A. Gábris, P. P. Rohde, K. Laiho, M. Štefaňák, V. Potoček, C. Hamilton, I. Jex, and C. Silberhorn, Science 336, 55 (2012). 
[21] N. Inui, Y. Konishi, and N. Konno, Phys. Rev. A 69, 052323 (2004).

[22] K. Watabe, N. Kobayashi, M. Katori, and N. Konno, Phys. Rev. A 77, 062331 (2008).

[23] C. S. Hamilton, A. Gábris, I. Jex, and S. M. Barnett, New J. Phys. 13, 013015 (2011).

[24] C. Di Franco, M. Mc Gettrick, and Th. Busch, Phys. Rev. Lett. 106, 080502 (2011).

[25] C. Di Franco, M. Mc Gettrick, T. Machida, and Th. Busch, Phys. Rev. A 84, 042337 (2011).

[26] P. W. Anderson, Phys. Rev. 109, 1492 (1958).

[27] M. Mohseni, P. Rebentrost, S. Lloyd, and A. Aspuru-Guzik, J. Chem. Phys. 129, 174106 (2008).

[28] M. B. Plenio and S. F. Huelga, New J. Phys. 10, 113019 (2008).
[29] H. Obuse and N. Kawakami, Phys. Rev. B 84, 195139 (2011).

[30] D. Shapira, O. Biham, A. J. Bracken, and M. Hackett, Phys. Rev. A 68, 062315 (2003).

[31] J. Košík, V. Bužek, and M. Hillery, Phys. Rev. A 74, 022310 (2006).

[32] ImagEM, EM-CCD Camera C9100-14 (Hamamatsu), http://sales.hamamatsu.com/assets/pdf/hpspdf/e_c910013.pdf.

[33] S. Hisatake, K. Shibuya, and T. Kobayashi, Appl. Phys. Lett. 87, 081101 (2005).

[34] S. Hisatake, K. Tada, and T. Nagatsuma, Opt. Express 16, 21753 (2008).

[35] T. Schwartz, G. Bartal, S. Fishman, and M. Segev, Nature (London) 446, 52 (2007).

[36] P. Rebentrost, M. Mohseni, I. Kassal, S. Lloyd, and A. AspuruGuzik, New J. Phys. 11, 033003 (2009). 